\title{
Neural correlates of emotional processing in depression: Changes with cognitive behavioral therapy and predictors of treatment response
}

\author{
Maureen Ritchey $^{\mathrm{a}, \mathrm{b}, *, 1}$, Florin Dolcos ${ }^{\mathrm{c}, * *, 1}$, Kari M. Eddington ${ }^{\mathrm{d}, * * *, 1}$, Timothy J. Strauman ${ }^{\mathrm{b}}$, \\ Roberto Cabeza ${ }^{a, b}$ \\ ${ }^{a}$ Center for Cognitive Neuroscience, Duke University, Box 90999, LSRC Bldg. Durham, NC 27708, USA \\ ${ }^{\mathrm{b}}$ Department of Psychology \& Neuroscience, Duke University, Durham, NC, USA \\ ${ }^{c}$ Department of Psychology and Beckman Institute for Advanced Science and Technology, University of Illinois at Urbana-Champaign, Urbana, IL, USA \\ ${ }^{\mathrm{d}}$ Department of Psychology, The University of North Carolina at Greensboro, Greensboro, NC, USA
}

\section{A R T I C L E I N F O}

\section{Article history:}

Received 23 June 2010

Received in revised form

24 August 2010

Accepted 11 September 2010

\section{Keywords:}

Event-related fMRI

Depression

Mood disorders

Affective disorders

Cognitive therapy

Affect

\begin{abstract}
A B S T R A C T
Major depressive disorder (MDD) is characterized by the presence of disturbances in emotional processing. However, the neural correlates of these alterations, and how they may be affected by therapeutic interventions, remain unclear. The present study addressed these issues in a preliminary investigation using functional magnetic resonance imaging (fMRI) to examine neural responses to positive, negative, and neutral pictures in unmedicated MDD patients $(N=22)$ versus controls $(N=14)$. After this initial scan, MDD patients were treated with cognitive behavioral therapy (CBT) and scanned again after treatment. Within regions that showed pre-treatment differences between patients and controls, we tested the association between pre-treatment activity and subsequent treatment response as well as activity changes from pre- to post-treatment. This study yielded three main findings. First, prior to treatment and relative to controls, patients exhibited overall reduced activity in the ventromedial prefrontal cortex (PFC), diminished discrimination between emotional and neutral items in the amygdala, caudate, and hippocampus, and enhanced responses to negative versus positive stimuli in the left anterior temporal lobe (ATL) and right dorsolateral PFC. Second, CBT-related symptom improvement in MDD patients was predicted by increased activity at baseline in ventromedial PFC as well as the valence effects in the ATL and dorsolateral PFC. Third, from pre- to post-treatment, MDD patients exhibited overall increases in ventromedial PFC activation, enhanced arousal responses in the amygdala, caudate, and hippocampus, and a reversal of valence effects in the ATL. The study was limited by the relatively small sample that was able to complete both scan sessions, as well as an inability to determine the influence of comorbid disorders within the current sample. Nevertheless, components of the neural networks corresponding to emotion processing disturbances in MDD appear to resolve following treatment and are predictive of treatment response, possibly reflecting improvements in emotion regulation processes in response to $\mathrm{CBT}$.
\end{abstract}

(c) 2010 Elsevier Ltd. All rights reserved.

\footnotetext{
* Corresponding author. Duke University, Center for Cognitive Neuroscience, Box 90999, LSRC Bldg. Durham, NC 27708, USA. Tel.: +1 919668 2299; fax: +1 919681 0815.

** Corresponding author. University of Illinois at Urbana-Champaign, 405 North Mathews Ave, Urbana, IL, 61801, USA. Tel.: +1 2172444120.

*** Corresponding author. The University of North Carolina at Greensboro, PO Box 26170, Greensboro, NC 27402, USA. Tel.: +1 3362560569.

E-mail addresses: maureen.ritchey@duke.edu (M. Ritchey), fdolcos@uiuc.edu (F. Dolcos), kmedding@uncg.edu (K.M. Eddington).

1 Shared first authorship.
}

\section{Introduction}

A key feature of major depressive disorder (MDD) is the presence of disturbances in emotional processing, which generally are expressed as a negative bias in processing emotional information (e.g., Gotlib et al., 2005; Koster et al., 2005; Siegle et al., 2002a). Specifically, patients with MDD tend to experience increased negative affect and reduced positive affect, and these mood disturbances are accompanied by negative affective biases during the perception and interpretation of emotional information. Patients with MDD show attentional biases toward cues for sadness or dysphoria (Gotlib et al., 2004) and tend to interpret neutral or positive information 
negatively compared to nondepressed individuals (Gollan et al., 2008; Gur et al., 1992). However, a number of questions remain about the mechanisms underlying these alterations in the way MDD patients process emotional information, and how such mechanisms may be affected by therapeutic interventions.

One avenue for understanding the neural substrates of MDD has been to explore how the brain instantiates the observed biases in emotional processing. There have been a wide variety of efforts to characterize neural differences between patients with MDD and healthy controls, interrogating either resting state or task-related differences between groups with an emphasis on established emotional processing networks. These approaches have revealed functional disturbances in specific brain regions, such as the medial prefrontal cortex (PFC; Price and Drevets, 2009), particularly the anterior cingulate cortex (ACC), as well as in the amygdala (AMY) and other limbic regions (see Drevets, 2001 for a review).

The medial PFC appears to serve at least two distinct purposes with regard to emotion processing (Bush et al., 2000). Ventromedial PFC (vmPFC) and ventral ACC (vACC), including subgenual and pregenual ACC, are thought to be part of an emotion-sensitive network that includes the AMY and increases activity following exposure to emotionally-salient stimuli (Bush et al., 2000; Phillips et al., 2003). Dorsomedial PFC (dmPFC) and dorsal ACC (dACC), including supragenual ACC, have been associated with cognitive control processes that, in the context of emotion processing, serve to regulate emotionrelated responses in the ventral network (Phillips et al., 2003). In addition, pregenual ACC has been posited to facilitate communication between more ventral and dorsal sectors of the PFC (Mayberg, 1997). In general, patients with MDD tend to exhibit enhanced activity within vmPFC/vACC and reduced activity within dmPFC/ dACC (Fitzgerald et al., 2008; Matthews et al., 2008; Mayberg, 1997). However, this pattern has not been entirely consistent across studies. Other evidence points to a decrease in vACC activity in patients with MDD (Drevets et al., 1997; Elliott et al., 2002; Lee et al., 2008), possibly due to a reduction in cortical volume in this area (Drevets, 2001; Drevets et al., 1997). Interpretation of these findings is further complicated by the wide variability in imaging methods, task designs, and patient characteristics (e.g., number of previous episodes, treatment history), as well as by the functional heterogeneity of the medial frontal regions.

Findings in the AMYalso have been mixed. It has been shown that AMY metabolism during the resting state is elevated in depressed patients (Drevets et al., 1992), consistent with a pattern of AMY hyper-reactivity in patients with MDD. Also, in tasks involving presentation of negative and neutral material, patients tend to show exaggerated AMY responses to negative (relative to neutral or positive) material (Fales et al., 2008; Hamilton and Gotlib, 2008; Siegle et al., 2002b), consistent with a negativity bias. However, other reports indicate that AMY responses are elevated for both negative and neutral material (Almeida et al., 2010; Sheline et al., 2001 ), or not elevated at all relative to healthy controls (Davidson et al., 2003). Nevertheless, there appears to be some consensus concerning alterations in the AMY's functions associated with depression.

Recent investigations also have attempted to delineate the interaction of these neural differences with various forms of treatment. One important question is whether the neural differences between MDD patients and nondepressed controls persist after treatment, or whether successful treatment eliminates or reduces such differences. The vast majority of studies addressing this question have used pharmacological antidepressant treatments, and typically report normalization of pre-treatment activity differences in both cortical regions, including dmPFC/dACC and vmPFC/vACC (Fitzgerald et al., 2008; Mayberg et al., 1999), as well as subcortical structures, including AMY (Anand et al., 2007; Fales et al., 2009; Fu et al., 2004; Sheline et al., 2001).

It remains unclear, however, whether these changes in patterns of neural activation are specific to pharmacological treatments, which may suggest a specific mechanism of action, or whether similar changes are observed for non-pharmacological interventions. A handful of studies have compared groups of patients treated with antidepressants to those treated with brief, structured psychotherapies. In comparisons of antidepressant medication and interpersonal psychotherapy (IPT) (Brody et al., 2001; Martin et al., 2001), Brody et al. (2001) found that both forms of treatment yielded similar effects on the brain: increased resting-state metabolism in the insula and the inferior temporal regions and decreased metabolism in the lateral PFC, vACC, and caudate, with the effects moving in the direction of normalization. Similarly, Martin et al. (2001) found only limited differences between patients treated with medications versus IPT: the antidepressanttreated group exhibited increased resting-state metabolism in right lateral posterior temporal cortex, whereas the therapy group had increased metabolism in right posterior cingulate cortex. Neither study reported any treatment-related changes in the AMY (Brody et al., 2001; Martin et al., 2001).

Other studies have examined the effects of treatment with cognitive behavioral therapy (CBT), an approach that emphasizes challenging and restructuring depressed patients' negative cognitions (Hollon et al., 2002). Compared to pharmacological antidepressants, this form of therapy may reflect a more "topdown" approach to resolving depressive symptoms (Goldapple et al., 2004; Simons et al., 1984). In one study, depressed patients treated with CBT showed increased resting-state metabolism in hippocampus and dorsal mid-cingulate, but reduced metabolism in dorsolateral, ventrolateral, and medial PFC regions (Goldapple et al., 2004). However, this pattern was not found in patients treated with antidepressants, thus suggesting distinct mechanisms of change associated with CBT (Goldapple et al., 2004). Unlike the aforementioned studies of therapy effects on neural activity, which employed resting-state designs rather than emotion-related tasks, a recent study examined CBT influences on neural responses during an implicit facial affect processing task. Comparisons of pre- versus post-treatment activity revealed that task-related elevations in AMY activity were reduced post-CBT, and in contrast to the results of Goldapple et al., mid/dorsal ACC activity increased after treatment (Fu et al., 2008). Finally, there is additional evidence that CBT modulates brain activity in patients with anxiety disorders, which are frequently comorbid with MDD. For example, after CBT, phobic patients show reductions in hyperactivation of the dorsolateral PFC (Paquette et al., 2003) and the dorsal ACC (Straube et al., 2006) in response to fear-relevant stimuli.

Despite progress in elucidating treatment-related changes in brain activity, a number of important questions concerning the effect of treatment on the neural correlates of emotion processing in MDD remain. For instance, the influence of CBT on neural activity associated with recovery from depression remains largely unspecified, mainly due to the paucity of research on this issue and the lack of consistency in available findings. Furthermore, these investigations have only rarely incorporated an assessment of neural differences between depressives and controls associated with emotion processing, which may help to elucidate some of the core features of MDD. Another unresolved issue is whether baseline neural responsivity, particularly to emotionally-salient stimuli, may predict subsequent treatment outcome. Improving the prediction of subsequent treatment response is an important goal of research on MDD (Kemp et al., 2008), and neuroimaging data may provide useful measures for these assessments. To the extent that neural 
"markers" can be used to predict differential response to medication or psychotherapy, such prospective analyses may contribute to the development of treatment matching strategies. Antidepressant-related symptom improvement has been shown to correlate with pre-treatment activity in the ACC, extending from dACC (Chen et al., 2007; Davidson et al., 2003) to pregenual (Mayberg et al., 1997) and subgenual ACC (Chen et al., 2007), such that greater pre-treatment activations at baseline predicted greater symptom improvement. A few studies have identified predictors of CBT response, likewise implicating ACC regions, although the localization and directionality of these results have been inconsistent (Fu et al., 2008; Siegle et al., 2006). For example, Fu et al. (2008) demonstrated that prior to treatment, valence modulation in the dorsal ACC was reduced in subsequent treatment responders versus non-responders, resulting in activation patterns that were comparable between responders and healthy controls. The results of Siegle et al. (2006), on the other hand, showed a relationship between treatment-related improvement and reduced pre-treatment activity in the subgenual ACC. In addition to the difference in localization, in this case participants showing the greatest improvement differed the most from healthy controls. The studies also differed with respect to whether amygdala activity predicted CBT response, with one study linking heightened pre-treatment amygdala activity to subsequent improvement (Siegle et al., 2006) and other showing no relationship (Fu et al., 2008). Given these varied results, the identification of predictors of $\mathrm{CBT}$ response merits further exploration.

In sum, although there is evidence that the neural correlates of depression are sensitive to treatment with CBT and predict treatment outcome, the pattern of these interactions has not yet been consistently characterized. The present study unites these experimental questions within the context of an emotion processing task and a pre- and post-therapy experimental design, thereby allowing us to track emotion-specific activation patterns associated with depression as well as their relationship to CBT within a single study. Unmedicated patients with MDD were scanned using functional magnetic resonance imaging (fMRI) while viewing negative, positive, and neutral pictures during two scan sessions, before and after CBT treatment, and compared with a group of nondepressed controls scanned during the same task. Because of the novelty of this approach, we view this study as a preliminary investigation into these issues. Three main indices of emotion processing were evaluated: overall activity (negative, neutral, and positive collapsed together), arousal-related activity (negative and positive $>$ neutral), and valence-related activity (negative versus positive). For each of these orthogonal contrasts, multiple analysis strategies were employed to subserve three main goals. First, we aimed to characterize neural differences between patients with MDD and controls before treatment. Second, we sought to identify which of these pre-treatment differences are predictive of treatment outcome. Third, we assessed which of these pre-treatment differences are mitigated after CBT.

\section{Methods}

\subsection{Participants}

Participants in the first scan session included patients with current $\operatorname{MDD}(N=22 ; 59.1 \%$ female $)$ and controls with no personal or family history of affective disorder $(N=14 ; 64.3 \%$ female), who were matched on age and gender to the first 14 enrolled depressed patients. Depressed patients ranged in age from 21 to $56(M=36.1$, $\mathrm{SD}=10.1)$, and controls ranged from 24 to $44(M=34.6, \mathrm{SD}=6.9)$. Out of the initially-scanned patients, 15 (60\% female) completed treatment and returned for the second scan session; functional data from the second scan was lost due to technical error for 4 of these participants, leaving 11 MDD participants (72.7\% female) with intact functional data from both scans. Out of the initiallyscanned control participants, 7 (71.4\% female) returned for the second scan session. The low return rate among controls was due to upgrades in the scanner facilities that prevented completion of session 2 for the remaining control participants. Therefore, data from the control group's second session were excluded from the fMRI analyses.

Depressed patients met DSM-IV criteria for current major depressive disorder (MDD) of at least moderate severity. Most of the patients were diagnosed with recurrent MDD (77\%) while the remaining $23 \%$ met criteria for a single episode. Of those patients with recurrent MDD, 10 reported having between 1 and 5 previous episodes and 7 reported 6 or more. Exclusion criteria included history of mania or psychotic symptoms, borderline or antisocial personality disorder, and current substance dependence. Comorbid Axis I diagnoses, including anxiety disorders, were acceptable as long as the current depressive episode was primary. Eight patients met criteria for a comorbid anxiety disorder ( 1 social phobia, 4 generalized anxiety disorder, 1 panic disorder, 1 obsessive-compulsive disorder, and 1 post-traumatic stress disorder) and one met criteria for comorbid cannabis abuse. Depression severity was moderate on average, with a mean BDI score of $25.1(\mathrm{SD}=8.8)$ and a mean 17 -item HRSD of 26.7 (SD = 6.7).

Exclusion criteria for both groups included the following: lefthanded or ambidextrous, history of neurological disorder or head trauma, evidence of cognitive impairment, pregnancy, and any implanted metal or other medical devices/conditions that were contraindicated with MRI. A pre-requisite for recruitment was that participants in the depressed group had to be free of any antidepressant medications (including herbal remedies or antidepressants used for other indications) for at least 2 months prior to entering the study. Potential control group participants who reported a personal or family history of any affective disorder or who met criteria for any current Axis I psychopathology (except for simple phobia) were excluded. All participants gave informed consent prior to participation in the study.

\subsection{Procedures}

Depressed and control participants were scanned while performing an emotion evaluation task during two separate sessions. The first MRI scanning session took place between 1 and 2 weeks following the initial evaluation. After the first scan, the patients received a standard course of individual CBT. Once they completed this course of treatment, patients returned for another functional scan, during which they performed the same emotion evaluation task with a novel set of stimuli. The BDI was administered both prior to the initial scan and after treatment. This study design was approved by institutional review board of Duke University Medical Center.

\subsection{Pre-scan assessments}

Participants in the depressed group were interviewed by a clinician using the Structured Clinical Interview for the DSM-IV (SCID; First et al., 1995) and the Hamilton Rating Scale for Depression (HRSD; Hamilton, 1960). Participants then completed the Beck Depression Inventory (BDI; Beck et al., 1961), a widelyused self-report measure of depressive symptom severity. Both scales have been shown to have good internal consistency and reliability. Participants in the depressed group were required to have a minimum BDI score of 17 to qualify for the study. Participants in the control group were interviewed using the nonpatient 
version of the SCID (First et al., 1995). Known family history (in the immediate family, including parents, biological siblings, and offspring) of any affective disorder was also assessed in this group via participant self-report.

\subsection{Treatment}

Patients received a naturalistic course of individual CBT, following Beck's (1995) cognitive therapy manual and using worksheets from the Mind Over Mood workbook (Greenberger and Padesky, 1995). Sessions were $50 \mathrm{~min}$ in length and were scheduled once per week. The three therapists (including one of the authors, KME) were all Ph.D.-level clinical psychologists who had a minimum of five years of experience with CBT.

Length of treatment was based on two factors, completion of the key components of CBT and symptom change. All patients received a full course of $C B T$ prior to termination, including identifying and challenging negative automatic thoughts, conducting behavioral experiments, and identifying and challenging negative core beliefs. Upon completion of the key CBT components, treatment was ended for patients who maintained BDI scores in the nonclinical range (0-13) for at least four consecutive sessions. Patients who completed the key CBT components but had not yet maintained clinically significant improvement remained in treatment either until that criterion had been met or until gains had reached a plateau, which was the case for one participant. ${ }^{2}$ Length of treatment averaged 20.7 sessions ( $S D=7.6$; range $10-35$ ) and 30.3 weeks $(\mathrm{SD}=12.5$; range $10-49)$ among the participants who returned for the post-treatment scan session. CBT resulted in significant improvement in patients' depressive symptoms from pre- to posttreatment. For those participants who completed both scan sessions, post-treatment BDI scores $(M=4.4, \mathrm{SD}=5.7)$ were significantly lower than pre-treatment scores $(M=23.0, \mathrm{SD}=8.7 ; t(14)=6.20$, $p<0.001)$. Clinically significant change was defined according to previously established BDI score norms (Seggar et al., 2002), requiring that patients' BDI scores change by at least 8 points in addition to having a score of 14 or less. Twelve out of the 15 MDD patients (80\%) who returned for the second scan session showed clinically significant improvement under these criteria.

\subsection{Stimuli}

Each participant was presented with 90 positive, 90 negative, and 90 neutral pictures selected from the International Affective Picture System (IAPS) picture database (Lang et al., 2001), on the basis of their normative arousal and valence scores. The mean IAPS arousal scores $(1=$ calm, $9=$ excited $)$ were 6.03 for positive $(\mathrm{SD}=2.18), 6.06$ for negative $(\mathrm{SD}=2.2$ ), and 3.06 for neutral pictures $(S D=1.95)$. Thus, positive and negative pictures had similar arousal scores, whereas neutral pictures had low arousal scores. The mean valence scores $(1=$ negative, $5=$ neutral, $9=$ positive) were 7.14 for positive ( $S D=1.61)$, 2.39 for negative $(\mathrm{SD}=1.51)$, and 5.03 for neutral $(\mathrm{SD}=1.29)$. Males and females viewed slightly different sets of stimuli due to expected sex differences in response to specific images; these differences mainly reflected changes in the sex of the characters depicted in the positive pictures with sexual content. The above scores reflect the average of these sets. To equate the emotional and neutral

\footnotetext{
${ }^{2}$ Only patients who successfully completed treatment were invited to participate in the second scan session. This stringent criterion may account for what appears to be a high drop-out rate between sessions 1 and 2 among the patient group. In fact, 5 of the 7 patient "drop-outs" completed 6-8 weeks of CBT, which under other criteria, may have qualified them as treatment completers.
}

categories for visual complexity and content (e.g., human presence), the IAPS pictures were supplemented with neutral pictures from other sources (Yamasaki et al., 2002).

\subsection{Emotion evaluation task}

The pool of 270 pictures was divided into 9 sets of 30 pictures (10 positive, 10 negative, and 10 neutral), which were randomly assigned to 9 blocks. Three of these blocks were presented during the first session (pre-treatment), and the other 6 were presented during the second session (post-treatment). Thus, different sets of pictures were assigned to each session, without repetition. To balance the number of stimuli between the first and second sessions, only the first 3 blocks from the second session were included in the present analyses. Participants were randomly assigned to one of six different block orders. To avoid the induction of long-lasting mood states, the pictures within each block were pseudo-randomized so that no more than two pictures of the same valence were consecutively presented. Functional MR images were recorded while subjects viewed emotional and neutral pictures. The pictures were presented, using an LCD projector, to a screen located behind the subjects' crown that subjects could see via an angled mirror. Each picture was presented for $3 \mathrm{~s}$ and followed by a 12-s fixation cross. Participants were instructed to experience any feelings or thoughts the pictures might elicit in them, and to then rate each picture in a 3 -point pleasantness scale $(1=$ unpleasant, $2=$ neutral, 3 = pleasant) using a button box in the scanner (Dolcos et al., 2004a,b). Responses were not speeded, although participants were asked to respond while the pictures were on-screen if possible. Thus, responses could occur at any time and response times reflect time after picture onset.

\subsection{Behavioral data analysis}

Affect ratings were collected from 18 MDD patients and 9 control participants during the first scan session, and from 6 MDD patients during the second scan session. Ratings from the other participants were lost due to technical or experimenter error. Average ratings and response times from the pre-treatment session were submitted to a 2 (Group: MDD, control) $\times 3$ (Emotion type: negative, neutral, positive) mixed ANOVA. Average ratings from both sessions were additionally submitted to 2 (Session: pretreatment, post-treatment) $\times 3$ (Emotion type: negative, neutral, positive) repeated-measures ANOVA, for the subset of patients with intact behavioral data from both scan sessions.

\section{8. fMRI data acquisition and analysis}

For both scan sessions, images were acquired on a GE Signa 1.5-T scanner (Waukesha, Wisconsin). Functional T2*-weighted images sensitive to the blood-oxygenation-level-dependent (BOLD) contrast were acquired using a spiral gradient-echo sequence $(\mathrm{TR}=2000 \mathrm{~ms}, \mathrm{TE}=40 \mathrm{~ms}$, flip angle $=90$, matrix $=64 \times 64$; inplane resolution $=3.9 \mathrm{~mm}^{2}$ ). The functional imaging volume consisted of 28 contiguous $4-\mathrm{mm}$ slices acquired in an interleaved fashion parallel to the line connecting the anterior and posterior commissures. Prior to functional acquisition, a T1-weighted structural set including a 28-slice image coplanar with the functional was acquired for coregistration.

The first and second scan sessions for each participant were processed and modeled independently. The functional data were pre-processed and analyzed in SPM2 (http://www.fil.ion.ucl.ac.uk/ spm), implemented in Matlab. Data were corrected for head motion, resliced to a resolution of $3.75 \times 3.75 \times 5 \mathrm{~mm}$, normalized to the MNI template, and smoothed with an $8 \mathrm{~mm}$ kernel. Motion 
Table 1

Behavioral data.

\begin{tabular}{|c|c|c|c|c|c|c|}
\hline & \multicolumn{3}{|l|}{ Ratings } & \multicolumn{3}{|c|}{ Response Times (ms) } \\
\hline & Negative & Neutral & Positive & Negative & Neutral & Positive \\
\hline Control & $1.12(0.11)$ & $2.08(0.13)$ & $2.60(0.24)$ & $1794(332)$ & $1858(298)$ & $2045(382)$ \\
\hline MDD pre-TX & $1.21(0.16)$ & $2.04(0.14)$ & $2.58(0.33)$ & $1803(352)$ & $2007(436)$ & 1951 (388) \\
\hline MDD post-TX & $1.21(0.17)$ & $2.07(0.20)$ & $2.65(0.20)$ & $1926(646)$ & $2189(665)$ & 1853 (335) \\
\hline
\end{tabular}

Note. Ratings were collected on a 3 -point scale, with $1=$ unpleasant, $2=$ neutral, and $3=$ pleasant. Data reported as Mean (SD). TX $=$ treatment.

regressors were included as a nuisance covariate in the analysis; however, runs with excessive head movement (greater than $3 \mathrm{~mm}$ ) were excluded from analysis, with a maximum of 1 run excluded per participant. Regressors were included for negative, neutral, and positive trial types, separately for the pre-treatment and posttreatment fMRI data. Three orthogonal contrasts were evaluated at the subject level, indexing overall event-related effects (all trial types collapsed versus implicit baseline), arousal effects (negative and positive versus neutral), and valence effects (negative versus positive).

For each of these contrasts, multiple analytical strategies were used to subserve the three main goals of our investigation. First, to characterize neural differences between patients with MDD $(N=22)$ and controls $(N=14)$ before treatment, two-sample $t$-tests were employed for each of the three contrast types above, at $p<0.005$ and an extent threshold of 5 contiguous voxels. A slightly more liberal statistical threshold was appropriate given the preliminary nature of the study as well as the likelihood of substantial individual and trial-wise variability (Lieberman and Cunningham, 2009). Second, to identify which of these pretreatment differences were predictive of treatment outcome, across-subject voxel-wise correlations were calculated between pre-treatment contrasts in brain activity in MDD patients $(N=15)$ and individual measures of improvement (i.e., percent improvement in BDI scores from pre- to post-treatment). Third, to further assess which of the pre-treatment differences between depressives and controls were mitigated in the depressed group after CBT, voxel-wise paired-sample $t$-tests were performed by comparing the patient group's pre-treatment contrasts to posttreatment contrasts $(N=11)$. The latter two analyses were restricted to voxels that fell within functionally-defined ROIs that were generated based on the pre-treatment comparisons between the MDD and control groups subserving the first goal. These functional ROIs were defined as $10 \mathrm{~mm}$ spheres around the peak voxels identified in a limited set of regions by the pre-treatment group comparisons. The voxel-wise tests subserving the second and the third goals conducted within these functional ROIs used an intensity threshold of $p=0.05$ and an extent threshold of 5 voxels. Thus, the voxels identified by this procedure not only showed the effect of interest, but also resided within regions showing pre-treatment group differences. Although the small number of controls completing both sessions impeded our ability to perform a full group by time interaction, as in Davidson et al. (2003), the pre- to post-treatment comparisons in the patient group were exclusively masked (at $p=0.05$, a conservative threshold for exclusive masking) with the corresponding session comparisons in the control group, to mitigate concerns that these effects could be driven by repeated testing or the passage of time.
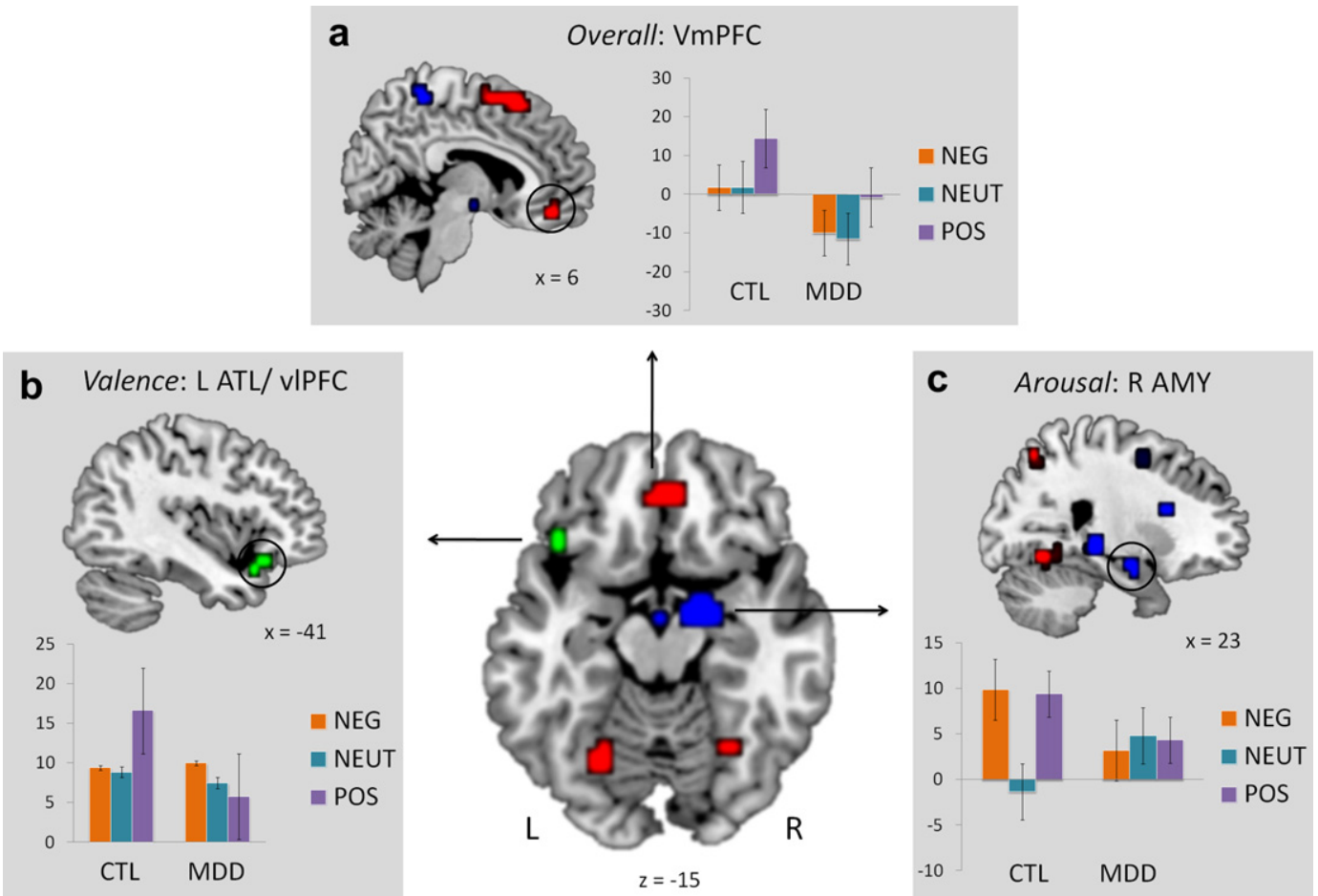

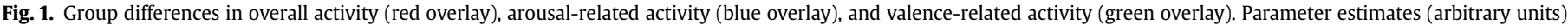

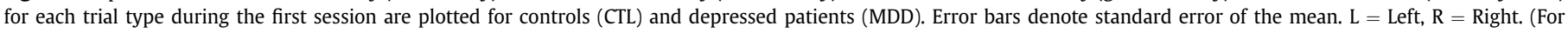
interpretation of the references to colour in this figure legend, the reader is referred to the web version of this article.) 
Table 2

Pre-Treatment Differences.

\begin{tabular}{|c|c|c|c|c|c|c|c|}
\hline \multirow[t]{2}{*}{ Region } & \multicolumn{7}{|c|}{ Talairach Coordinates } \\
\hline & BA & Hem & $x$ & $y$ & $z$ & $t$ & voxels \\
\hline \multicolumn{8}{|c|}{ Overall Activity (Negative, Neutral, \& Positive): Control $>$ MDD, $p<0.005$} \\
\hline VmPFC & 11 & & 4 & 43 & -15 & 3.55 & 10 \\
\hline Superior Frontal Gyrus & 6 & $\mathrm{R}$ & 4 & 6 & 55 & 3.5 & 36 \\
\hline Fusiform Gyrus & 19 & $\mathrm{R}$ & 26 & -59 & -5 & 3.73 & 11 \\
\hline Superior Parietal Lobule & 7 & $\mathrm{R}$ & 26 & -63 & 49 & 3.72 & 5 \\
\hline Lingual Gyrus & 19 & $\mathrm{~L}$ & -22 & -62 & -9 & 3.64 & 15 \\
\hline Cuneus & 17 & $\mathrm{~L}$ & -7 & -80 & 4 & 3.66 & 17 \\
\hline \multicolumn{8}{|c|}{$\begin{array}{l}\text { Arousal-Related Activity (Negative \& Positive }>\text { Neutral): Control }>\text { MDD, } \\
\quad p<0.005\end{array}$} \\
\hline Amygdala & & $\mathrm{R}$ & 19 & -4 & -12 & 5.55 & 13 \\
\hline Caudate Nucleus & & $\mathrm{R}$ & 19 & 12 & 18 & 3.84 & 9 \\
\hline Hippocampus & & $\mathrm{L}$ & -26 & -26 & -3 & 3.72 & 8 \\
\hline Hippocampus & & $\mathrm{R}$ & 26 & -29 & -3 & 3.65 & 17 \\
\hline DIPFC & 6 & $\mathrm{R}$ & 30 & 6 & 50 & 3.17 & 7 \\
\hline DIPFC & 6 & $\mathrm{~L}$ & -33 & 3 & 55 & 4.07 & 12 \\
\hline Mid-Cingulate Gyrus & 24 & $\mathrm{~L}$ & -4 & -5 & 37 & 3 & 5 \\
\hline Superior Temporal Gyrus & 39 & $\mathrm{~L}$ & -52 & -58 & 12 & 3.49 & 9 \\
\hline Paracentral Lobule & 4 & $\mathrm{R}$ & 7 & -37 & 57 & 3.82 & 6 \\
\hline Superior Parietal Lobule & 7 & $\mathrm{R}$ & 15 & -62 & 63 & 3.31 & 5 \\
\hline \multicolumn{8}{|c|}{ Valence-Related Activity (Negative $>$ Positive): MDD $>$ Control, $p<0.005$} \\
\hline $\begin{array}{l}\text { Anterior Temporal } \\
\text { Lobe/Ventrolateral PFC* }\end{array}$ & 38 & $\mathrm{~L}$ & -45 & 17 & -18 & 3.91 & 14 \\
\hline DIPFC* & 6 & $\mathrm{R}$ & 63 & -9 & 33 & 3.61 & 14 \\
\hline Insula* & & $\mathrm{R}$ & 41 & -17 & 24 & 3.6 & 9 \\
\hline Dorsal ACC & 32 & $\mathrm{~L}$ & -4 & 34 & 31 & 3.72 & 25 \\
\hline VIPFC & 45 & $\mathrm{R}$ & 48 & 25 & -5 & 3.44 & 15 \\
\hline Superior Frontal Sulcus & 9 & $\mathrm{~L}$ & -30 & 23 & 31 & 3.43 & 5 \\
\hline Medial Frontal Gyrus & 6 & $\mathrm{~L}$ & -4 & -1 & 55 & 3.34 & 9 \\
\hline VIPFC & 6 & $\mathrm{~L}$ & -45 & -2 & 42 & 3.92 & 13 \\
\hline Insula & & $\mathrm{L}$ & -33 & 8 & 4 & 3.27 & 6 \\
\hline Precentral Gyrus & 4 & $\mathrm{~L}$ & -22 & -19 & 61 & 4.2 & 13 \\
\hline Precentral Gyrus & 4 & $\mathrm{~L}$ & -7 & -29 & 66 & 3.76 & 5 \\
\hline Hippocampus & & $\mathrm{L}$ & -19 & -26 & -7 & 3.44 & 7 \\
\hline Superior Temporal Gyrus & 22 & $\mathrm{~L}$ & -48 & -26 & -3 & 3.92 & 11 \\
\hline Inferior Temporal Gyrus & 37 & $\mathrm{~L}$ & -45 & -51 & -6 & 3.62 & 5 \\
\hline Superior Temporal Gyrus & 39 & $\mathrm{~L}$ & -52 & -58 & 12 & 4.06 & 11 \\
\hline Inferior Parietal Lobule & 40 & $\mathrm{R}$ & 59 & -34 & 43 & 3.32 & 5 \\
\hline Precuneus & 7 & $\mathrm{R}$ & 11 & -45 & 44 & 3.52 & 8 \\
\hline Middle Occipital Gyrus & 19 & $\mathrm{~L}$ & -41 & -73 & 4 & 4.41 & 28 \\
\hline Fusiform Gyrus & 19 & $\mathrm{~L}$ & -30 & -80 & -4 & 4 & 5 \\
\hline
\end{tabular}

Note. ${ }^{*}$ denotes regions from the valence comparison that remained after restricting the comparison to only those regions showing negative $>$ positive in the MDD group. $\mathrm{BA}=$ Brodmann Area, Hem = Hemisphere, $\mathrm{L}=$ Left, $\mathrm{R}=$ Right.

\section{Results}

\subsection{Behavioral results}

Average ratings and response times are reported in Table 1. There was a main effect of emotion type on ratings from the first session, $F(2,50)=329.05, p<0.001$, in that negative items were rated as more unpleasant and positive items more pleasant, but no main effect of group, $F(1,25)=0.04, p=0.84$, or interaction of emotion type and group, $F(2,50)=0.86, p=0.43$. This pattern confirms that, for both groups, there was concordance between the emotion labels and their perception of the pictures. Ratings from the MDD patients were additionally examined for the effect of session. There was a main effect of emotion type, $F(2,10)=52.48$, $p<0.001$, but no main effect of session, $F(1,5)=1.24, p=0.32$. There was also a marginal interaction between emotion type and session, $F(2,10)=3.33, p=0.08$, reflecting a modest shift toward higher ratings for the positive pictures after treatment.

There was also a main effect of emotion type on response times from the first session, $F(2,50)=5.14, p=0.01$, in that response times increased as item valence increased, but we detected no main effect of group, $F(1,25)=0.03, p=0.88$, or interaction of emotion type and group, $F(2,50)=1.86, p=0.17$. Response times from the MDD patients were additionally examined for the effect of session. There were no observed main effects of emotion type, $F(2,10)=2.25, p=0.16$, or session, $F(1,5)=0.20, p=0.67$. There was a marginal interaction between emotion type and session, $F(2,10)=4.04, p=0.052$, reflecting a modest shift toward slower response times for negative and neutral pictures and faster response times for positive pictures after treatment.

\subsection{Functional MRI results}

\subsubsection{Pre-treatment differences}

Pre-treatment group differences were evaluated by comparing MDD patients to controls on three main contrasts: overall activity, arousal-related activity, and valence-related activity (Fig. 1 and Table 2). For overall activity (all trials > implicit baseline), group differences were identified in the vmPFC and regions in parietal and visual cortex, with controls showing greater activity in these regions than patients. The reduction in vmPFC activity in MDD patients (see Fig. 1a) is consistent with previous findings of disrupted processing in this region associated with MDD (Drevets et al., 1997; Elliott et al., 2002; Lee et al., 2008). No gray matter a

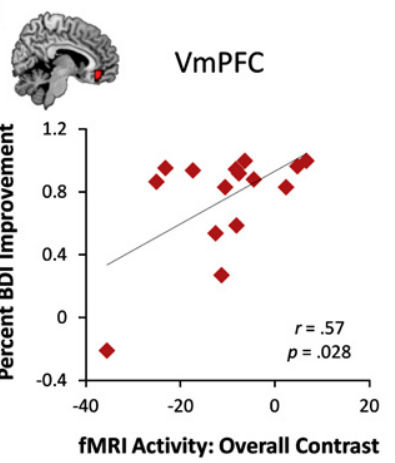

b
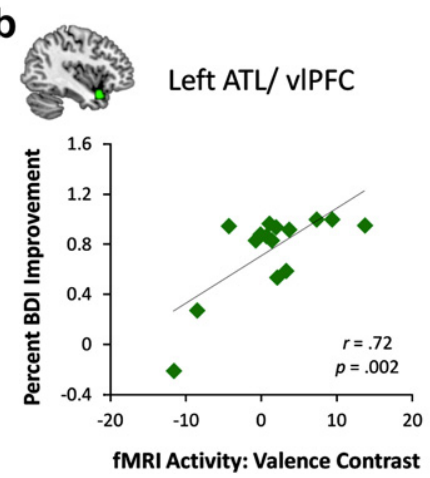

C

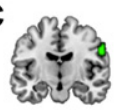

Right dIPFC

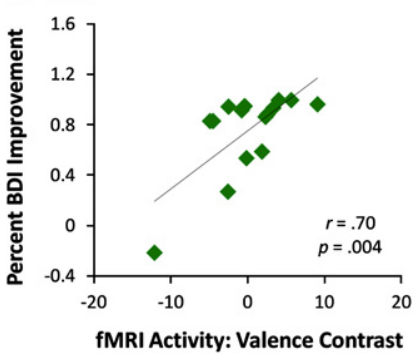

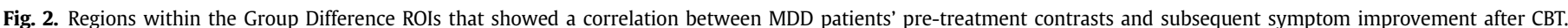

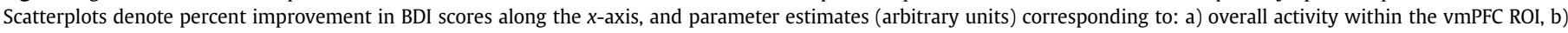

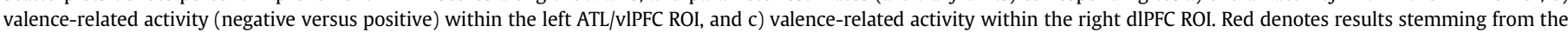

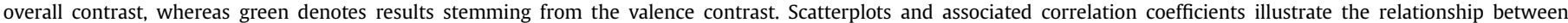

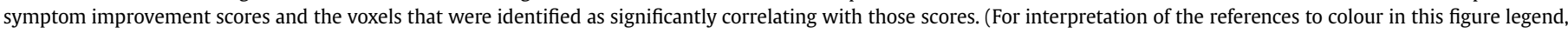
the reader is referred to the web version of this article). 
a
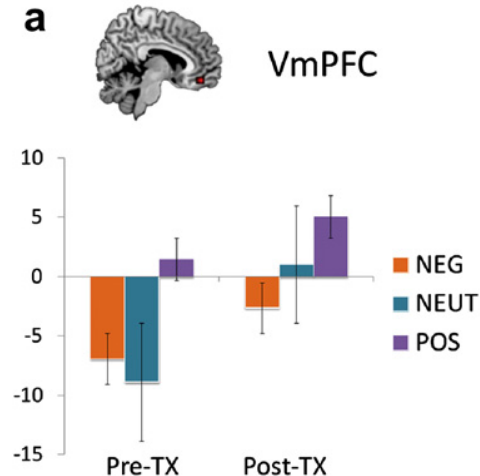

b

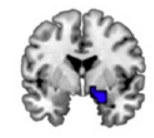

Right AMY

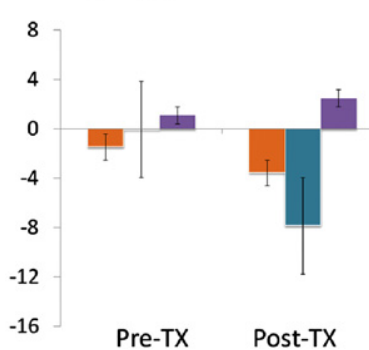

C
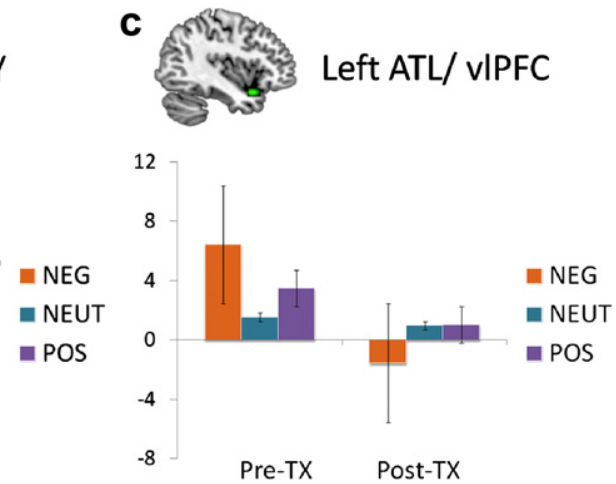

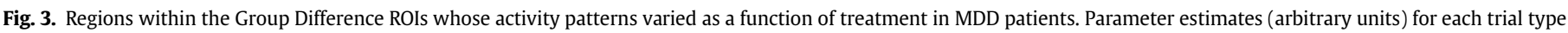

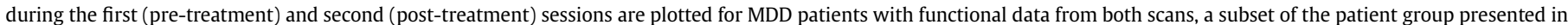
Fig. 1. Error bars denote standard error of the mean.

regions exhibited the reverse pattern, although one white matter cluster emerged near the posterior parahippocampal gyrus (Talairach: $-37,-43,2$ ).

For arousal-related activity (negative and positive $>$ neutral), group differences were identified in the right AMY, right caudate, and bilateral hippocampus, among other regions (Table 2). These differences reflected a greater difference between emotional and neutral stimuli in the control group than in the patient group. That is, whereas activation in the right AMY discriminated emotional and neutral items in the controls, such activation did not discriminate item types in the patients (see Fig. 1c).

Finally, for valence-related activity (negative $>$ positive), while group differences were identified across a wide variety of regions (see Table 2), most of these differences were driven by greater activity to positive than negative in the control group. When we restricted these regions to those also showing greater activity to negative than positive in the patient group, consistent with a negativity bias, only three clusters met this criterion: the right insula, the right dlPFC, and a cluster appearing to span both the left anterior temporal lobe (ATL) and vlPFC (BA 47). Both dlPFC and vlPFC have been previously linked with emotion regulation processes (Ochsner et al., 2002; Ochsner and Gross, 2008), which may be differentially engaged in response to negative versus positive material. Although we cannot discern whether the ATL/vlPFC cluster originates from ATL, vlPFC, or both, these regions have both been associated with semantic elaboration (Bookheimer, 2002; Martin and Chao, 2001), and the vlPFC in particular may participate in regulatory processing (Aron et al., 2004; Dolcos et al., 2006).

\subsubsection{Pre-treatment predictors of treatment response}

Brain-behavior correlation analyses restricted to voxels from the functional ROIs identified in selected regions showing pretreatment differences revealed clusters whose pre-treatment activation patterns predicted subsequent treatment response (Fig. 2). Specifically, for the overall contrast, a cluster within the vmPFC ROI (Fig. 2a) positively correlated with symptom improvement, indicating that although patients generally showed reduced activity in this region, those patients with higher levels of activity (i.e., more similar to controls) were more likely to respond to CBT. For the arousal contrast, no regions within the AMY, caudate, or hippocampal ROIs correlated with treatment response. Finally, valence-related activity within the left ATL (Fig. 2b) and right dIPFC (Fig. 2c) positively correlated with symptom improvement, suggesting that patients who exhibited the strongest negativity bias in these regions also tended to improve the most with CBT.

\subsubsection{Changes from pre- to post-treatment}

These same ROIs also were examined for treatment-related changes by comparing the patients' pre- to post-treatment responses (Fig. 3). For the overall contrast, a cluster within the vmPFC ROI significantly increased from pre- to post-treatment, although at 4 voxels, the cluster size was slightly smaller than our specified extent threshold. The right AMY, right caudate, and left hippocampal ROIs included clusters exhibiting session effects for the arousal contrast, evidenced by a larger difference between emotional versus neutral trials post-treatment compared to pretreatment. Finally, valence-related activity in left ATL was additionally modulated by session: this region showed a negativity bias in patients before treatment, but afterwards exhibited a positivity bias similar to that in pre-treatment controls.

\section{Discussion}

The present study provides new evidence that the neural differences between depressed patients and healthy controls during emotion processing are sensitive to cognitive behavioral therapy, with a subset of affected regions predicting treatment outcome and normalizing after therapeutic intervention. The study yielded three main findings. First, prior to treatment, group differences in activation patterns were identified in several regions. Relative to controls, patients exhibited overall reduced activity in the ventromedial vmPFC, diminished discrimination between emotional and neutral items in the AMY, caudate, and hippocampus, and enhanced responses to negative versus positive stimuli in the left ATL/vlPFC and right dIPFC. Second, pre-treatment activity in a subset of these regions additionally predicted CBTrelated improvement in MDD patients - increased activity in vmPFC as well as the negativity biases in left ATL and right dIPFC predicted greater symptom improvement. Third, activity in several of these regions was modulated by CBT. Specifically, from pre- to post-treatment, MDD patients exhibited overall increases in vmPFC activation, enhanced discrimination of emotional and neutral items in the amygdala and caudate, and greater activity in response to positive versus neutral items in the left ATL. Thus, among the identified pre-treatment differences between the patient and control groups, several regions were predictive of patients' treatment response and their activation was modulated by treatment.

\subsection{Pre-treatment differences}

The present results are consistent with previous studies reporting baseline neural activity differences between patients with MDD versus healthy controls. One frequently reported set of 
regions includes the VmPFC and VACC, where baseline group differences have been characterized as both activity increases (Fitzgerald et al., 2008; Mayberg, 1997) and activity decreases (Drevets et al., 1997; Elliott et al., 2002; Lee et al., 2008). The present findings also reflect group differences in vmPFC, consistent with a pre-treatment reduction in $\mathrm{vmPFC}$ activity, localized anterior to subgenual ACC. This reduction spanned all trial types, suggesting an overall trend toward diminished processing in this region in contexts requiring emotion evaluation.

We additionally identified pre-treatment group differences that varied by emotional arousal and valence. Although MDD patients did not exhibit an overall increase in AMYactivity before treatment, they demonstrated similarly high AMY responses to neutral as well as emotional material, in contrast to the emotion-specific response in controls. This finding complements recent evidence that depression is associated with amygdala hyper-responsivity to mild sad and neutral stimuli in particular, though that study was restricted to individuals with bipolar disorder (Almeida et al., 2010). This pattern of activity is consistent with a pattern of hyper-responsivity in the AMY. With respect to valence, although patients and controls rated the pictures similarly on our three-point scale, the use of neuroimaging data allows us to examine the neural substrates of emotional processing that may be more sensitive to valence processing shifts. To this end, we sought regions that were more active for negative than positive stimuli in patients but not in controls. The left ATL/vlPFC and right dIPFC exhibited this pattern, responding more to negative than positive pictures in patients but not controls.

These pre-treatment differences during an emotion processing task may be interpreted in light of evidence from emotion regulation studies, which involve a network of regions including vmPFC, vlPFC, and dlPFC (Ochsner et al., 2002; Ochsner and Gross, 2008). Emotion regulation strategies such as reappraisal are effective in reducing negative affect in healthy controls (Gross, 1998; Ochsner and Gross, 2008), a process thought to depend on prefrontallymediated down-regulation of arousal responses in the AMY (Ochsner et al., 2002; Wager et al., 2008). Depressed individuals fail to engage these prefrontal-AMY networks during emotion regulation (Johnstone et al., 2007), and score lower than controls on a scale indexing how much they use reappraisal strategies (Abler et al., 2007). This lack of reappraisal correlates with depression severity and predicts higher AMY responses during negative picture anticipation (Abler et al., 2007). Consistent with an emotion regulation account, $\mathrm{vmPFC}$ is involved in the consolidation of extinction learning (Quirk et al., 2000), and participates in cortical control of arousal responses in the AMY during extinction and emotion regulation (Delgado et al., 2008). Thus, overall pre-treatment reductions in this region may reflect patients' decreased ability to regulate emotional responses. In light of this interpretation, one avenue for future research would be to investigate the relationship between the vmPFC and amygdala as a function of CBT in MDD. Prior work has demonstrated a diminished correlation between activity in these regions in depressed patients (Anand et al., 2005a; Matthews et al., 2008), interpreted as reflecting decreased regulatory communication and feedback between cortical and limbic regions. Furthermore, the strength of coupling between pregenual ACC and AMY has been shown to increase as a function of treatment with pharmacological antidepressants (Anand et al., 2007, 2005b; Chen et al., 2008), raising the question of whether that pattern extends to treatment with CBT.

The findings that left ATL/vlPFC and right dIPFC exhibit a negativity bias in depressed patients before treatment may also be interpreted as perturbations in regulatory processing, or in semantic elaboration processes that support regulation. The left ATL and vIPFC have been associated with semantic elaboration (Bookheimer, 2002; Martin and Chao, 2001), and the vlPFC has been more specifically linked with inhibitory regulation processes (Dolcos et al., 2006). Furthermore, both vlPFC and dlPFC are associated with reappraisal processes (Ochsner et al., 2002), although there is evidence that in depressed patients reappraisal-related activations in these regions are not associated with diminished amygdala response to negative material (Johnstone et al., 2007). These previous findings suggest the possibility that the neural pattern of negative bias in left vIPFC/ATL and right dIPFC may reflect counterproductive engagement of regulatory processes during negative evaluation. DIPFC in particular has been previously linked with neural changes in depression, typically demonstrating hypoactivation (Fales et al., 2008; Siegle et al., 2007), although some studies have reported increased recruitment of dlPFC during tasks that involve executive control (Harvey et al., 2005; Walter et al., 2007). The present results expand this finding to include variation by emotional valence, with increased recruitment during negative evaluation but decreased during positive evaluation.

\subsection{Pre-treatment predictors of treatment response}

In addition to identifying regions displaying pre-treatment differences between MDD patients and healthy controls, we were interested in evaluating the degree to which CBT influences neural activity in these regions. One open question is whether pre-treatment activations in response to different types of affectively salient stimuli would be predictive of subsequent symptom improvement with CBT. Identifying predictors of treatment response is an essential goal of MDD research, as improvements in response prediction will facilitate the development of individualized treatment plans, saving time and suffering relative to trial-and-error methods (Kemp et al., 2008). Neuroimaging data, particularly that collected during emotion processing tasks (Kemp et al., 2008), may inform response prediction. Similar to studies linking pre-treatment activity to antidepressant efficacy (Mayberg et al., 1997), investigations using CBT have identified ACC activity as a critical predictor of symptom improvement, although these findings have been mixed. In one study, CBT-related improvements were greatest in patients with relatively low pre-treatment reactivity in subgenual ACC in response to emotional stimuli, compared to healthy controls, and the reverse occurred for AMY reactivity (Siegle et al., 2006). Another study linked increases in CBT-related improvement with reduced valence modulation in dorsal ACC, right dIPFC, and vlPFC - similar to the pattern in healthy controls (Fu et al., 2008). No significant relationship was identified with amygdala activity. Thus, while in the former study greater pre-treatment deviation from controls predicted better clinical outcomes for the patients, in the latter less pre-treatment deviation predicted better outcomes. The studies also differed with respect to localization within the ACC (i.e., subgenual), as well as the presence of a significant link between amygdala activity and symptom improvement.

The present results diverge from these previous findings. In the present study, overall activity in the vmPFC positively correlated with reduction in depressive symptoms, suggesting that patients with the least pre-treatment impairment in this region benefitted most from CBT. Thus, contrary to both of these previous studies, greater improvement was associated with increases, not decreases, in activity in vmPFC (near pregenual ACC, localized between subgenual and dorsal ACC); however, like Fu et al. (2008), this pattern reflected greater similarity between healthy controls and patients with the best subsequent outcome. The association in the present study is consistent with an emotion regulation account of the data. CBT incorporates a number of reappraisal strategies, which involve challenging one's interpretation of emotional stimuli and events. Patients who have higher vmPFC activity before treatment may have available the functional circuitry necessary to effectively use 
reappraisal strategies. This interpretation also is consistent with suggestions that CBT may capitalize on a patient's existing strengths, rather than compensating for deficits (Rude and Rehm, 1991). It should be noted that in the present study, arousalrelated activity in the amygdala did not predict subsequent CBT-related improvement, as has been previously reported (Siegle et al., 2006; but see Fu et al., 2008), leaving open the question of under which conditions this relationship emerges.

Valence-related activity in both left ATL/vlPFC and right dIPFC also predicted subsequent treatment response. In these regions, the strength of the negativity bias predicted the degree to which patients responded to CBT. This finding may seem counterintuitive since, in this case, patients who were most different from controls before treatment benefitted most from CBT. However, if these activation patterns reflect counterproductive attempts to regulate, it may be these patients who benefit most from the strategies learned during CBT. Alternatively, it may be that patients with heightened pre-treatment neural responsivity (represented by greater overall activity or greater bias) tend to improve most with treatment, which may account for both sets of positive correlations described here. This pattern is consistent with findings that heightened physiological responses (e.g., heart rate) before treatment predict the efficacy of exposure therapy (Beckham et al., 1990; Lang et al., 1970). Because of the variability between previous findings and those observed in the present study, it is prudent to reiterate that task-related differences across studies may account in part for the variability in findings. Furthermore, it is unlikely that any single methodology will provide the sensitivity and specificity required to apply response prediction to the clinical domain, which may additionally account for this variability. It has been suggested that the combination of multiple methodologies, including clinical, cognitive, neuroimaging, and genetic measures, may prove more effective in predicting treatment outcomes (Gudayol-Ferré et al., in press; Kemp et al., 2008). Although the present study was limited to identifying markers of response to CBT only, the positive results reported here and previously (Siegle et al., 2006; Fu et al., 2008) are suggestive of the possible merits of including neuroimaging data from emotion processing tasks as a component of future response prediction assessments.

\subsection{Changes from pre- to post-treatment}

Finally, the present study tested whether or not activity in regions showing pre-treatment differences changed as a function of treatment with CBT. Several of these regions showed a pattern of change consistent with the direction of normalization, including overall activity in vmPFC and valence-related activity in left ATL/vlPFC. These results may be interpreted as reflecting increased engagement of processes involved in modulating responses to affect-laden stimuli.

Interestingly, although pre-treatment differences in the AMY and caudate were not predictive of subsequent treatment outcome, these regions also changed as a function of CBT treatment. After treatment, these regions distinguished between emotional and neutral items, no longer responding to neutral items with the same magnitude of activation. One possible interpretation of these results is that activation within these subcortical structures may reflect essential responses to emotional arousal that are symptomatic of depression, but do not reflect processes that are predictive of CBT efficacy.

\subsection{Caveats}

While the present study was able to clarify neural correlates of depression and predictors and consequences of CBT, it was characterized by several limitations. As a preliminary investigation into the effects of CBT on emotion processing in MDD, this study's main limitation is a lack of power, with relatively small sample sizes of participants completing both fMRI scans. This impeded our ability to look at the full interaction of group with time, an effective method for distinguishing treatment and repeated testing effects (Davidson et al., 2003). Nevertheless, the results of this study may serve as an indication of the fruitfulness of this avenue of research and motivate future research on a larger scale. Larger sample sizes would also afford the ability to determine the extent to which emotion processing is modulated by comorbid disorders, such as anxiety, and how CBT differentially impacts these disorders. Although we interpret our results within the framework of emotion regulation processes, which CBT is commonly conceptualized to target, we did not experimentally manipulate the use of specific regulation strategies within the emotion processing task, although some degree of regulation is likely to have occurred naturally. Furthermore, the present emotion effects are limited to those elicited in the presence of both valence and arousal, since our negative and positive picture sets were highly arousing. An interesting direction for future research would be to use either nonarousing valenced stimuli or neutral interesting stimuli, since these manipulations may be able to more carefully disentangle the contributions of valence versus arousal. Additionally, because patients were treated until remission, the present study may have reduced variability in treatment response relative to other studies that used a strictly predefined number of treatment sessions. Finally, because we used CBT as our only treatment, we cannot evaluate the specificity of the results to CBT. Future studies that directly contrast different forms of treatment will be necessary to elucidate specific versus general effects of treatments for depression.

\section{Conclusions}

In summary, the present study tested the hypothesis that the neural correlates of depression would be sensitive to an emotion processing task, and that patterns of neural responses to emotionally-salient stimuli among depressed individuals would additionally predict clinical response to a subsequent CBT intervention. The data demonstrated that hypoactivation of the vmPFC and hyper-responsivity of the AMY change in the direction of normalization after CBT, although only vmPFC effects were predictive of treatment-related improvement. Likewise, valence effects patterned like negativity biases in depressed patients predicted patients' response to CBT, but not all of these effects reversed after treatment. Collectively, by uniting these analytic approaches within a single design, the present study sheds light on the dynamic nature of pre-treatment differences during the course of depression and recovery.

\section{Conflicts of interest}

All authors declare that they have no conflicts of interest.

\section{Contributors}

F.D., K.M.E., T.S., and R.C. designed the research, F.D. and K.M.E. performed the research, M.R. contributed new analytic tools, M.R., F.D., and K.M.E. analyzed the data, and all authors contributed to and have approved the final manuscript. 


\section{Role of funding source}

This work was supported by a grant from the Beck Institute for Cognitive Therapy and Research, by NIMH grant \# MH067447 to TJS, and by NINDS grant \# NS41328 to RC. FD was supported by a Young Investigator Award from the National Alliance for Research on Schizophrenia and Depression and a Research Award from the Canadian Psychiatric Research Foundation. MR was supported by a National Research Service Award \#F31MH085384. KE was supported by a National Research Service Award \# 5F32MH64261. None of the funding sources had any further role in study design; in the collection, analysis and interpretation of data; in the writing of the report; and in the decision to submit the paper for publication.

\section{References}

Abler B, Erk S, Herwig U, Walter $\mathrm{H}$. Anticipation of aversive stimuli activates extended amygdala in unipolar depression. Journal of Psychiatric Research 2007; 41:511-22.

Almeida JR, Versace A, Hassel S, Kupfer DJ, Phillips ML. Elevated amygdala activity to sad facial expressions: a state marker of bipolar but not unipolar depression. Biological Psychiatry 2010;67:414-21.

Anand A, Li Y, Wang Y, Gardner K, Lowe MJ. Reciprocal effects of antidepressant treatment on activity and connectivity of the mood regulating circuit: an fMRI study. Journal of Neuropsychiatry and Clinical Neurosciences 2007;19:274-82.

Anand A, Li Y, Wang Y, Wu J, Gao S, Bukhari L, et al. Activity and connectivity of brain mood regulating circuit in depression: a functional magnetic resonance study. Biological Psychiatry 2005a;57:1079-88.

Anand A, Li Y, Wang Y, Wu JW, Gao SJ, Bukhari L, et al. Antidepressant effect on connectivity of the mood-regulating circuit: an fMRI study. Neuropsychopharmacology 2005b;30:1334-44.

Aron AR, Robbins TW, Poldrack RA. Inhibition and the right inferior frontal cortex. Trends in Cognitive Sciences 2004;8:170-7.

Beck A, Ward C, Mendelson M, Mock J, Erbaugh J. An inventory for measuring depression. Archives of General Psychiatry 1961;4:561-71.

Beck J. Cognitive therapy: basics and beyond. New York, NY: Guilford Press; 1995.

Beckham JC, Vrana SR, May JG, Gustafson DJ, Smith GR. Emotional processing and fear measurement synchrony as indicators of treatment outcome in fear of flying. Journal of Behavior Therapy and Experimental Psychiatry 1990;21:153-62.

Bookheimer S. Functional MRI of language: new approaches to understanding the cortical organization of semantic processing. Annual Review of Neuroscience 2002;25:151-88.

Brody AL, Saxena S, Stoessel P, Gillies LA, Fairbanks LA, Alborzian S, et al. Regional brain metabolic changes in patients with major depression treated with either paroxetine or interpersonal therapy: preliminary findings. Archives of General Psychiatry 2001;58:631-40.

Bush G, Luu P, Posner MI. Cognitive and emotional influences in anterior cingulate cortex. Trends in Cognitive Sciences 2000:4:215-22.

Chen $\mathrm{CH}$, Ridler K, Suckling J, Williams S, Fu CHY, Merlo-Pich E, et al. Brain imaging correlates of depressive symptom severity and predictors of symptom improvement after antidepressant treatment. Biological Psychiatry 2007;62:407-14.

Chen $\mathrm{CH}$, Suckling J, Ooi C, Fu CH, Williams SCR, Walsh ND, et al. Functional coupling of the amygdala in depressed patients treated with antidepressant medication. Neuropsychopharmacology 2008;33:1909-18.

Davidson RJ, Irwin W, Anderle MJ, Kalin NH. The neural substrates of affective processing in depressed patients treated with venlafaxine. American Journal of Psychiatry 2003;160:64-75.

Delgado MR, Nearing KI, LeDoux JE, Phelps EA. Neural circuitry underlying the regulation of conditioned fear and its relation to extinction. Neuron 2008;59: 829-38.

Dolcos F, Kragel P, Wang LH, McCarthy G. Role of the inferior frontal cortex in coping with distracting emotions. Neuroreport 2006;17:1591-4.

Dolcos F, LaBar KS, Cabeza R. Dissociable effects of arousal and valence on prefrontal activity indexing emotional evaulation and subsequent memory: an eventrelated fMRI study. Neurolmage 2004a;23:64-74.

Dolcos F, LaBar KS, Cabeza R. Interaction between the amygdala and the medial temporal lobe memory system predicts better memory for emotional events. Neuron 2004b;42:855-63.

Drevets W. Neuroimaging and neuropathological studies of depression: implications for the cognitive-emotional features of mood disorders. Current Opinion in Neurobiology 2001;11:240-9.

Drevets WC, Price JL, Simpson JR, Todd RD, Reich T, Vannier M, et al. Subgenual prefrontal cortex abnormalities in mood disorders. Nature 1997;386:824-7.

Drevets WC, Videen T, Price J, Preskorn S, Carmichael S, Raichle M. A functional anatomical study of unipolar depression. Journal of Neuroscience 1992;12: 3628-41.

Elliott R, Rubinsztein JS, Sahakian BJ, Dolan RJ. The neural basis of mood-congruent processing biases in depression. Archives of General Psychiatry 2002;59: 597-604.
Fales CL, Barch DM, Rundle MA, Mintun MA, Mathews J, Snyder AZ, et al. Antidepressant treatment normalizes hypoactivity in dorsolateral prefrontal cortex during emotional interference processing in major depression. Journal of Affective Disorders 2009;112:206-11.

Fales CL, Barch DM, Rundle MM, Mintun MA, Snyder AZ, Cohen JD, et al. Altered emotional interference processing in affective and cognitive-control brain circuitry in major depression. Biological Psychiatry 2008;63:377-84.

First MB, Spitzer RL, Gibbon M, Williams JB. Structured clinical interview for DSMIV axis I disorders- patient edition (SCID-I/P, version 2.0). New York, NY: New York State Psychiatric Institute; 1995.

Fitzgerald PB, Laird AR, Maller J, Daskalakis ZJ. A meta-analytic study of changes in brain activation in depression. Human Brain Mapping 2008;29:683-95.

Fu CHY, Williams SCR, Cleare AJ, Brammer MJ, Walsh ND, Kim J, et al. Attenuation of the neural response to sad faces in major depression by antidepressant treatment - a prospective, event-related functional magnetic resonance imaging study. Archives of General Psychiatry 2004;61:877-89.

Fu CHY, Williams SCR, Cleare AJ, Scott J, Mitterschiffthaler MT, Walsh ND, et al. Neural responses to sad facial expressions in major depression following cognitive behavioral therapy. Biological Psychiatry 2008;64:505-12.

Goldapple K, Segal Z, Garson C, Lau M, Bieling P, Kennedy S, et al. Modulation of cortical-limbic pathways in major depression: treatment-specific effects of cognitive behavior therapy. Archives of General Psychiatry 2004;61:34-41.

Gollan JK, Pane HT, McCloskey MS, Coccaro EF. Identifying differences in biased affective information processing in major depression. Psychiatry Research 2008; $159: 18-24$

Gotlib IH, Krasnoperova E, Yue DN, Joormann J. Attentional biases for negative interpersonal stimuli in clinical depression. Journal of Abnormal Psychology 2004; 113:127-35.

Gotlib IH, Yue D, Joormann J. Selective attention in dysphoric individuals: the role of affective interference and inhibition. Cognitive Therapy and Research 2005;29: 417-32.

Greenberger D, Padesky C. Mind over mood: a cognitive therapy treatment manual for clients. New York, NY: Guilford Press; 1995.

Gross JJ. Antecedent- and response-focused emotion regulation: divergent consequences for experience, expression, and physiology. Journal of Personality and Social Psychology 1998;74:224-37.

Gudayol-Ferré E, Herrera-Guzmán I, Camarena B, Cortés-Penagos C, Herrera-Abarca JE, Martínez-Medina P, Cruz D, Hernández S, Genis A, Carrillo-Guerrero MY, Avilés Reyes R, Guàrdia-Olmos J. (2010). The role of clinical variables, neuropsychological performance and SLC6A4 and COMT gene polymorphisms on the prediction of early response to fluoxetine in major depressive disorder. Journal of Affective Disorders, doi:10.1016/j.jad.2010.06.002.

Gur RC, Erwin RJ, Gur RE, Zwil AS, Heimberg C, Kraemer HC. Facial emotion discrimination: II. Behavioral findings in depression. Psychiatry Research 1992;42:241-51.

Hamilton JP, Gotlib IH. Neural substrates of increased memory sensitivity for negative stimuli in major depression. Biological Psychiatry 2008:63:1155-62.

Hamilton M. A rating scale for depression. Journal of Neurology, Neurosurgery, and Psychiatry 1960;23:56-62.

Harvey P-O, Fossati P, Pochon J-B, Levy R, LeBastard G, Lehéricy S, et al. Cognitive control and brain resources in major depression: an fMRI study using the n-back task. NeuroImage 2005;26:860-9.

Hollon SD, Thase ME, Markowitz JC. Treatment and prevention of depression. Psychological Science in the Public Interest 2002;3:39-77.

Johnstone T, van Reekum CM, Urry HL, Kalin NH, Davidson RJ. Failure to regulate: counterproductive recruitment of top-down prefrontal-subcortical circuitry in major depression. Journal of Neuroscience 2007;27:8877-84.

Kemp AH, Gordon E, Rush AJ, Williams LM. Improving the prediction of treatment response in depression: integration of clinical, cognitive, psychophysiological, neuroimaging, and genetic measures. CNS Spectrums 2008;13: 1066-86.

Koster EHW, De Raedt R, Goeleven E, Franck E, Crombez G. Mood-congruent attentional bias in dysphoria: maintained attention to and impaired disengagement from negative information. Emotion 2005;5:446-55.

Lang PJ, Bradley MM, Cuthbert BN. International affective picture system (IAPS): instruction manual and affective ratings. Technical report A-5. Gainesville, FL: The Center for Research in Psychophysiology, University of Florida; 2001.

Lang PJ, Melamed BG, Hart J. A psychophysiological analysis of fear modification using an automated desensitization procedure. Journal of Abnormal Psychology 1970;76:220-34

Lee BT, Seok JH, Lee BC, Cho SW, Yoon BJ, Lee KU, et al. Neural correlates of affective processing in response to sad and angry facial stimuli in patients with major depressive disorder. Progress in Neuro-Psychopharmacology \& Biological Psychiatry 2008;32:778-85.

Lieberman MD, Cunningham WA. Type I and type II error concerns in fMRI research: re-balancing the scale. Social Cognitive \& Affective Neuroscience 2009;4:423-8.

Martin A, Chao LL. Semantic memory and the brain: structure and processes. Current Opinion in Neurobiology 2001;11:194-201.

Martin SD, Martin E, Rai SS, Richardson MA, Royall R. Brain blood flow changes in depressed patients treated with interpersonal psychotherapy or venlafaxine hydrochloride: preliminary findings. Archives of General Psychiatry 2001;58: 641-8.

Matthews SC, Strigo IA, Simmons AN, Yang TT, Paulus MP. Decreased functional coupling of the amygdala and supragenual cingulate is related to increased 
depression in unmedicated individuals with current major depressive disorder. Journal of Affective Disorders 2008;111:13-20.

Mayberg HS. Limbic-cortical dysregulation: a proposed model of depression. Journal of Neuropsychiatry and Clinical Neurosciences 1997;9:471-81.

Mayberg HS, Brannan SK, Mahurin RK, Jerabek PA, Brickman JS, Tekell JL, et al. Cingulate function in depression: a potential predictor of treatment response. Neuroreport 1997;8:1057-61.

Mayberg HS, Liotti M, Brannan SK, McGinnis S, Mahurin RK, Jerabek PA, et al Reciprocal limbic-cortical function and negative mood: converging PET findings in depression and normal sadness. American Journal of Psychiatry 1999;156: 675-82.

Ochsner KN, Bunge SA, Gross JJ, Gabrieli JDE. Rethinking feelings: an fMRI study of the cognitive regulation of emotion. Journal of Cognitive Neuroscience 2002;14:1215-29.

Ochsner KN, Gross JJ. Cognitive emotion regulation: insights from social cognitive and affective neuroscience. Current Directions in Psychological Science 2008; $17: 153-8$.

Paquette V, Lévesque J, Mensour B, Leroux J-M, Beaudoin G, Bourgouin P, et al "Change the mind and you change the brain": effects of cognitive-behaviora therapy on the neural correlates of spider phobia. NeuroImage 2003;18:401-9.

Phillips ML, Drevets WC, Rauch SL, Lane R. Neurobiology of emotion perception I: the neural basis of normal emotion perception. Biological Psychiatry 2003;54: 504-14.

Price JL, Drevets WC. Neurocircuitry of mood disorders. Neuropsychopharmacology 2009;35:192-216.

Quirk GJ, Russo GK, Barron JL, Lebron K. The role of ventromedial prefrontal cortex in the recovery of extinguished fear. Journal of Neuroscience 2000;20:6225-31.

Rude SS, Rehm LP. Response to treatments for depression: the role of initial status on targeted cognitive and behavioral skills. Clinical Psychology Review 1991;11:493-514.

Seggar LB, Lambert MJ, Hansen NB. Assessing clinical significance: application to the beck depression inventory. Behavior Therapy 2002;33:253-69.
Sheline YI, Barch DM, Donnelly JM, Ollinger JM, Snyder AZ, Mintun MA. Increased amygdala response to masked emotional faces in depressed subjects resolves with antidepressant treatment: an fMRI study. Biological Psychiatry 2001;50: $651-8$.

Siegle GJ, Carter CS, Thase ME. Use of fMRI to predict recovery from unipolar depression with cognitive behavior therapy. American Journal of Psychiatry 2006;163:735-8.

Siegle GJ, Ingram RE, Matt GE. Affective interference: an explanation for negative attention biases in dysphoria? Cognitive Therapy and Research 2002a;26: 73-87.

Siegle GJ, Steinhauer SR, Thase ME, Stenger VA, Carter CS. Can't shake that feeling: event-related fMRI assessment of sustained amygdala activity in response to emotional information in depressed individuals. Biological Psychiatry 2002b; 51:693-707.

Siegle GJ, Thompson W, Carter CS, Steinhauer SR, Thase ME. Increased amygdala and decreased dorsolateral prefrontal BOLD responses in unipolar depression: related and independent features. Biological Psychiatry 2007;61:198-209.

Simons AD, Garfield SL, Murphy GE. The process of change in cognitive therapy and pharmacotherapy for depression: changes in mood and cognition. Archives of General Psychiatry 1984;41:45-51.

Straube T, Glauer M, Dilger S, Mentzel H-J, Miltner WHR. Effects of cognitive-behavioral therapy on brain activation in specific phobia. NeuroImage 2006;29: 125-35.

Wager TD, Davidson ML, Hughes BL, Lindquist MA, Ochsner KN. Prefrontal-subcortical pathways mediating successful emotion regulation. Neuron 2008;59:1037-50.

Walter H, Wolf RC, Spitzer M, Vasic N. Increased left prefrontal activation in patients with unipolar depression: an event-related, parametric, performancecontrolled fMRI study. Journal of Affective Disorders 2007;101:175-85.

Yamasaki H, LaBar KS, McCarthy G. Dissociable prefrontal brain systems for attention and emotion. Proceedings of the National Academy of Sciences USA 2002;99:11447-51. 